\title{
Occlusion of the right subclavian artery after insertion of a transesophageal echocardiography probe in an adult with a vascular ring -A case report-
}

\author{
Sung Yong Park, Dae Hee Kim, Han Bum Joe, Sun Kyung Park, Ji Hoon Hwang, and Yong Woo Hong \\ Department of Anesthesiology and Pain Medicine, Ajou University of College of Medicine, Suwon, Korea
}

Intraoperative transesophageal echocardiography (TEE) has become an important monitoring device for patients undergoing cardiac or noncardiac surgery. Complications associated with TEE are unusual, but the potential for TEE probe compression of the posterior vascular structures has been reported in pediatric patients. We present here a case of occlusion of the right subclavian artery in an adult patient with a vascular ring after insertion of a TEE probe. (Korean J Anesthesiol 2010; 59: 283-285)

Key Words: Complications, Transesophageal echocardiography, Vascular ring.

The use of transesophageal echocardiogram (TEE) has recently become more prevalent in monitoring cardiac surgeries and surgeries involving possible severe hemodynamic change. Although complications associated with TEEs are very rare, and when they do occur they are due to the insertion of a probe $[1,2]$. In the present case, a TEE probe used on an adult patient with congenital right subclavian arterial mutation caused compression of the subclavian artery. Therefore, we evaluated related incidents and made a report of the case.

\section{Case Report}

A 75-year-old, $155 \mathrm{~cm}, 53 \mathrm{~kg}$ female patient presented for offpump coronary artery bypass graft surgery. As a preoperative test, a coronary angiography was performed. The results revealed $86 \%$ stenosis of the left anterior descending artery, $83 \%$ stenosis of the left circumflex artery and $91 \%$ stenosis of the right coronary artery. The aortography revealed that only the right common carotid artery was aberrant in the aortic arch. The branching of the right subclavian artery could not be checked (Fig. 1), but delayed imaging of the right subclavian artery was observed (Fig. 2). ECG and other tests did not reveal other abnormal findings and there were no symptoms of dysphagia or respiratory difficulty.

The patient was pre-operatively administered $6 \mathrm{mg}$ of morphine by muscular injection, was after which she was moved to the operating room. Five electrocardiogram leads were placed, and leads II and V5 were continuously monitored.

Received: December 17, 2009. Revised: January 2, 2010. Accepted: February 3, 2010.

Corresponding author: Yong Woo Hong, M.D., Department of Anesthesiology and Pain Medicine, Ajou University of College of Medicine, San 5, Woncheon-dong, Yeongtong-gu, Suwon 443-721, Korea. Tel: 82-31-219-5689, Fax: 82-31-219-5579, E-mail: ywhong7292@gmail.com (c) This is an open-access article distributed under the terms of the Creative Commons Attribution Non-Commercial License (http:// creativecommons.org/licenses/by-nc/3.0/), which permits unrestricted non-commercial use, distribution, and reproduction in any medium, provided the original work is properly cited. 


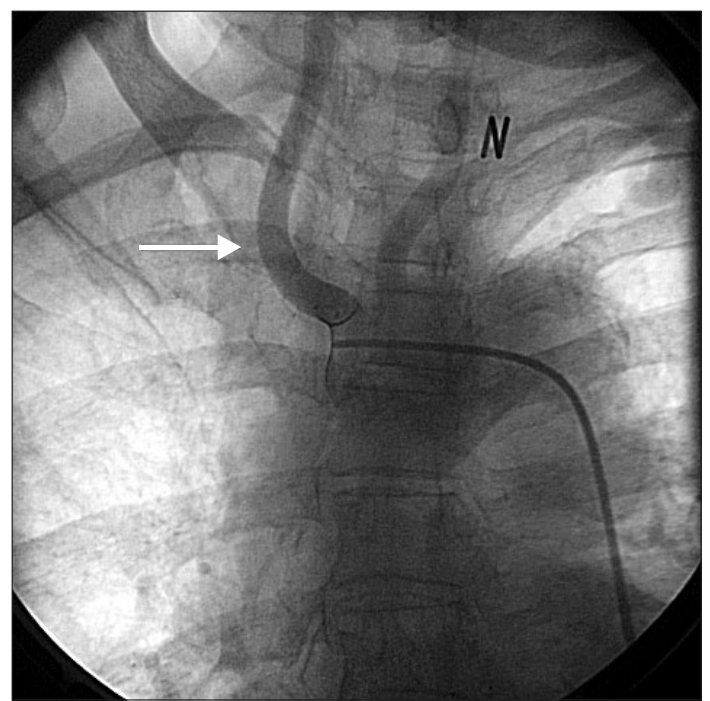

Fig. 1. Angiography of the aortic arch. The right common carotid artery (arrow) was branched from the aortic arch without the right subclavian artery showing.

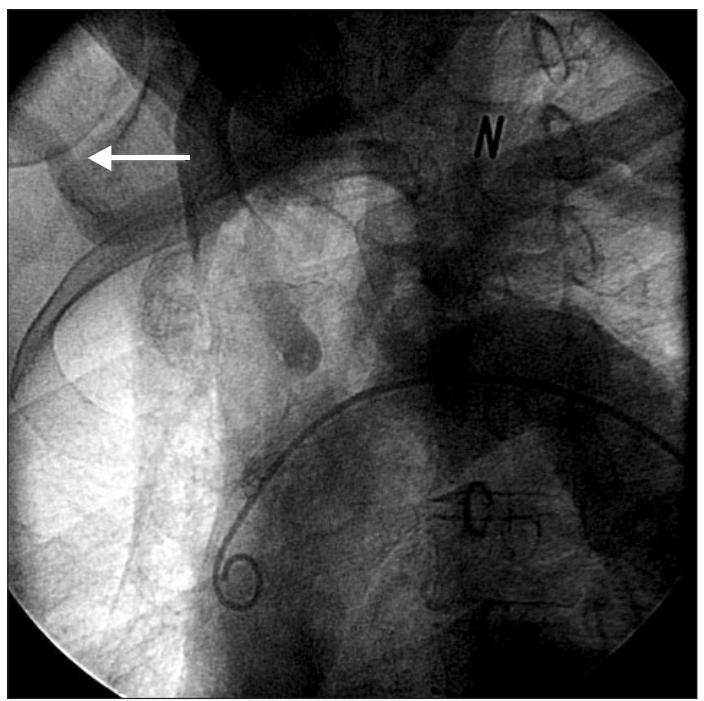

Fig. 2. Angiography of the aortic arch shows delayed visualization of the aberrant right subclavian artery (arrow).

The pulses of both radial arteries were palpated well and there was no difference in blood pressure between the two upper extremities. Because the left radial artery was going to be used as the graft vessel, a 20-gauge catheter was inserted into the right radial artery, and continuous monitoring of the arterial pressure was initiated. A pulmonary artery catheter was inserted into the right internal jugular vein to monitor cardiac performance parameters continuously.

To induce anesthesia, $50 \mu \mathrm{g}$ sufentanil, $2.5 \mathrm{mg}$ midazolam, and $10 \mathrm{mg}$ vecuronium were administered and endotracheal intubation was performed. Anesthesia was then maintained
A

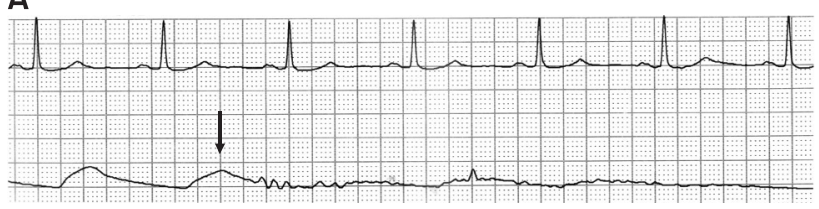

B

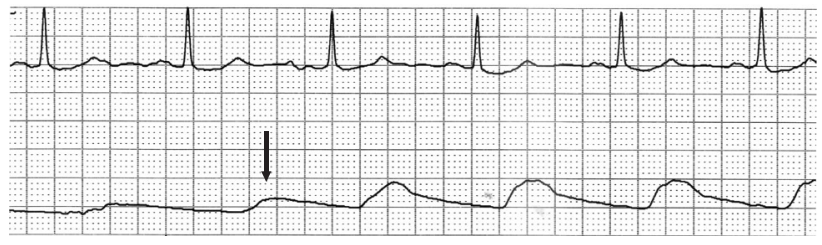

Fig. 3. Dampening of arterial waveforms by the transesophageal echocardiography (TEE) probe. Arrows indicate the beginning and end of the insertion of the TEE probe. (A) Beginning of the insertion of the TEE probe, (B) End of the insertion of the TEE probe.

using a continuous sufentanil infusion $(0.5-1.5 \mu \mathrm{g} / \mathrm{kg} / \mathrm{hr})$ and supplemental sevoflurane $(0.6-2.0$ vol\%). Next, a 20 -gauge catheter was inserted into the right femoral artery to enable accurate blood pressure monitoring. The radial artery pressure was monitored along with the continuous monitoring of the femoral artery pressure. Hemodynamic conditions following the induction of anesthesia were stable, and a $14 \times 12.5 \mathrm{~mm}$ TEE probe (6T probe, GE Vingmed Ultrasound, Horten, Norway) was inserted into the patient's esophagus. Immediately after insertion of the TEE probe, the arterial pressure waveforms of the right radial artery decreased significantly, whereas the right femoral artery pressure remained stable. The TEE probe was slowly removed from the patient's esophagus, after which the right radial waveform reappeared $28 \mathrm{~cm}$ from the upper incisor. After slowly advancing and retracting the probe twice, we observed the dampening of arterial pressure reoccur (Fig. 3). Based on these findings, it was suspected that the TEE probe caused compression of the right subclavian artery; therefore, the TEE probe was removed. Diluted blood was observed on the tip of the TEE probe after it was removed, indicating that the esophagus may have been damaged by the probe. Accordingly, a physician immediately conducted an esophageal endoscopy, which revealed an esophageal stenosis following pulsation on the posterior esophageal wall. With the use of the scope, we found the location of the stenosis to be $28 \mathrm{~cm}$ from the upper incisor, but no damage to the esophageal wall was observed. A $9.0 \times 9.0 \mathrm{~mm}$ gastroscope (GIF-XQ260, Olympus Medical System Co., Tokyo, Japan) was used for esophageal endoscopy and easily passed through the area of the stenosis. No compression of the right subclavian artery was observed; therefore, we inserted a $10.9 \times 8.4 \mathrm{~mm}$ pediatric TEE probe (9T probe, GE Vingmed Ultrasound, Horten, Norway) and proceeded with the surgery. No unusual conditions were observed during or after 
surgery.

\section{Discussion}

We experienced compression of the right subclavian artery caused by the TEE in an adult patient who had a vascular ring. Vascular rings are a rare congenital anomaly in which the fetal structure of the aortic arch persists [3]. Vascular rings account for less than $1 \%$ of all cardiopulmonary mutations and are grouped based on their anatomical characteristics [4]. The most common type of complete ring is a double aortic arch, which consists of two arches that connect posteriorly and form a vascular ring. A right aortic arch and right subclavian artery that is retropharyngeal with a left-sided ductus arteriosus is the next most common types of vascular rings. Vascular rings also include the pulmonary artery sling and the aberrant right subclavian artery. The most common incomplete rings are a retropharyngeal right subclavian artery, innominate artery compression, and a pulmonary artery sling $[4,5]$. Vascular rings compress the trachea and esophagus and cause respiratory difficulty or dysphagia. However, ECG and other tests cannot detect these symptoms; therefore, they often exist without the patient's knowing of their presence [4-7]. The patient in the present case had a right subclavian artery that was aberrant in the descending aorta of the distal portion of the left subclavian artery and passed between the esophagus and the vertebral body. In most cases, such variations do not present abnormal findings [7], and the patient in this case also had no symptoms prior to surgery.

TEEs have increasingly been used for anesthetic management in cardiac and noncardiac surgeries for patients with cardiac defects and other hemodynamic problems. Serious complications such as esophageal perforation, respiratory obstruction and ventilator failure may occur; however, such phenomena are rare. Accordingly, TEEs are relatively safe for use in patient management $[1,2]$. There have been reports of patients with vascular rings in which the TEE probe caused vascular compression, but these cases involved babies or young children for whom the probe was larger than their body surface area $[2,6,8,9]$. There are currently no other known cases of an adult who had the same complications as the patient described in the present case.

It is important to emphasize in this case that obstruction of the right subclavian artery for a long period of time can lead to ischemic damage and necrosis. When TEE is used for monitoring during surgery, as in the present case, if obstruction of the artery is not discovered early, the probe may cause vascular compression for a long period of time. Mechanical damage to the narrowed esophageal mucous membrane by the TEE probe can lead to hemorrhage, laceration, or perforation. Therefore, if resistance is felt when the TEE probe is inserted in patients with congenital anomalies one must refrain from inserting the probe by force. After insertion, it is necessary to compare the blood pressure of both upper extremities or to continuously monitor the other subclavian artery pressure or femoral artery pressure.

Here, we presented a case of occlusion of the right subclavian artery by a TEE probe in an adult patient with a vascular ring. Cases of vascular compression by the TEE probe in patients with vascular rings are rarer for adults than for children; nevertheless, they do occur in adults. Accordingly, careful attention must be taken with respect to monitoring methods and operative techniques when using a TEE probe.

\section{References}

1. Kallmeyer IJ, Collard CD, Fox JA, Body SC, Shernan SK. The safety of intraoperative transesophageal echocardiography: a case series of 7200 cardiac surgical patients. Anesth Analg 2001; 92: 1126-30.

2. Stevenson JG. Incidence of complications in pediatric transesophageal echocardiography: experience in 1650 cases. J Am Soc Echocardiogr 1999; 12: 527-32.

3. Bonnard A, Auber F, Fourcade L, Marchac V, Emond S, Révillon Y. Vascular ring abnormalities: a retrospective study of 62 cases. J Pediatr Surg 2003; 38: 539-43.

4. Rosen DA, Rosen KR. Anomalies of the aortic arch and valve. In: Pediatric Cardiac Anesthesia. 3rd ed. Edited by Lake CL: Stamford, Appletion \& Lange. 1998, pp 458-61.

5. Turner A, Gavel G, Coutts J. Vascular rings-presentation, investigation and outcome. Eur J Pediatr 2005; 164: 266-70.

6. Koinig H, Schlemmer M, Keznickl FP. Occlusion of the right subclavian artery after insertion of a transoesophageal echocardiography probe in a neonate. Paediatr Anaesth 2003; 13: 617-9.

7. Arciniegas E, Hakimi M, Hertzler JH, Farooki ZQ, Green EW. Surgical management of congenital vascular rings. J Thorac Cardiovasc Surg 1979; 77: 721-7.

8. Muhiudeen Russell IA, Miller-Hance WC, Silverman NH. Intraoperative transesophageal echocardiography for pediatric patients with congenital heart disease. Anesth Analg 1998; 87: 1058-76.

9. Bensky AS, O'Brien JJ, Hammon JW. Transesophageal echo probe compression of an aberrant right subclavian artery. J Am Soc Echocardiogr 1995; 8: 964-6. 